\title{
A large pseudo-Hall effect in n-type 3C-SiC(1 00 l $)$ and its dependence on crystallographic orientation for stress sensing applications
}

\author{
Afzaal Qamar $^{\mathrm{a}, *}$, Toan Dinh ${ }^{\mathrm{b}}$, Mohsen Jafari ${ }^{\mathrm{a}}$, Alan Iacopi ${ }^{\mathrm{b}}$, Sima Dimitrijev ${ }^{\mathrm{b}}$, Dzung Viet Dao ${ }^{\mathrm{b}}$ \\ ${ }^{a}$ Department of Electrical Engineering and Computer Science, University of Michigan, Ann Arbor, MI 48109, USA \\ ${ }^{\mathrm{b}}$ Queensland Micro- and Nanotechnology Centre, Griffith University, Queensland, Australia
}

\section{A R T I C L E I N F O}

\section{Keywords:}

Pseudo-Hall effect

3C-SiC

Piezoresistive effect

\begin{abstract}
A B S T R A C T
The pseudo-Hall effect in n-type single crystal 3C-SiC(1 00 ) with low carrier concentration has been investigated. Low pressure chemical vapor deposition was used to grow the single crystal n-type 3C-SiC( $\left.\begin{array}{lllllllll}1 & 0 & 0\end{array}\right)$ and Hall devices were fabricated by photolithography and dry etch processes. A large pseudo-Hall effect was observed in the grown thin films which showed a strong dependence on the crystallographic orientation. N-type 3C-SiC(1 0 0 $)$ with low carrier concentration shows a completely different behavior of pseudo-Hall measurements as compared to the p-type 3C-SiC(1 000$)$. Contrary to p-type, the effect is maximum along $\left[\begin{array}{llllllll}1 & 0 & 0\end{array}\right]$ crystallographic orientation and minimum along [ $\left.\begin{array}{lll}1 & 1 & 0\end{array}\right]$ orientation. Moreover, the observed pseudo-Hall effect is $50 \%$ larger than p-type with higher carrier concentration grown by the same process which makes n-type 3C-SiC( 100 ) with low carrier concentration more suitable material for designing highly sensitive micro-mechanical sensors.
\end{abstract}

\section{Introduction}

The dependence of the offset voltage of Hall devices (or four terminal devices) on applied stress can be utilized in stress sensing applications, thanks to the imperfections in the fabrication of Hall devices which generate this offset. Rather than taking it a reliability issue, this effect called pseudo-Hall effect can be useful for designing micro-mechanical sensors [1-4]. Compared to the two terminal diffused resistors, the pseudo-Hall effect offers many advantages which includes the elimination of Wheatstone bridge from the measuring circuitry, thermal stability and the small device size [5-8]. After reporting of this effect a number of studies were carried out on $\mathrm{Si}$ based devices and it has been shown that this effect can be used for stress, strain, pressure and force sensing in micro-electromechanical systems (MEMS) devices $[9,10]$. However, due to the low energy band gap of Si and its degradation in corrosive environment limits its use for high temperature and high radiation environment so that, other materials which are inert to radiation and have higher band gap needs to be explored. GaN, Diamond, GaAs and $\mathrm{SiC}$ are among those materials which can be used for harsh environment applications.
Among these materials, silicon carbide ( $\mathrm{SiC}$ ), with its large energy band, good chemical inertness and excellent mechanical properties, is an emerging potential material for applications used in harsh environments. $\mathrm{SiC}$ has various polytypes $2 \mathrm{H}-\mathrm{SiC}, 4 \mathrm{H}-\mathrm{SiC}, 6 \mathrm{H}-\mathrm{SiC}$ and $3 \mathrm{C}-\mathrm{SiC}$, however, only the $4 \mathrm{H}-\mathrm{SiC}, 6 \mathrm{H}-\mathrm{SiC}$, and $3 \mathrm{C}-\mathrm{SiC}$ are reliable semiconductors in the industry [6]. A number of studies has been focused on 3C-SiC for stress sensing applications [11-16]. 3C-SiC polytype has more advantage over other polytypes because it can be readily grown on commercially available large diameter Si substrates. Therefore, the cost of using homo-epitaxial films is significantly reduced if $\mathrm{Si}$ wafer is used for 3C-SiC thin films [17] compared to costly $4 \mathrm{H}-\mathrm{SiC}$ and $6 \mathrm{H}-\mathrm{SiC}$ substrates.

A number of studies on pseudo-Hall effect in p-type 3C-SiC have been reported recently, and it has been shown that the pseudo-Hall effect in p-type 3C-SiC( $\left.\begin{array}{lll}1 & 0 & 0\end{array}\right)$ varies significantly with applied stress/ strain showing its potential to be used for stress sensing applications. It has also been reported that the pseudo-Hall effect in p-type 3C-SiC strongly depends upon the crystal orientation and the device geometry [18-22]. However, in this study the pseudo-Hall effect in n-type 3C-SiC with low carrier concentration is presented. The influence of crystal orientation on the pseudo-Hall effect has also been investigated. The

\footnotetext{
* Corresponding author.

Email address: afzaal@umich.edu (A. Qamar)
} 
results achieved in this study are very useful for designing of highly sensitive MEMS stress/strain sensors from n-type 3C-SiC.

\section{Experimental}

Single crystal n-type 3C-SiC(1 00 ) thin films were grown to a thickness of $300 \mathrm{~nm}$ on $\mathrm{Si}\left(\begin{array}{lll}1 & 0 & 0\end{array}\right)$ substrate by LPCVD process at temperature of $\left(1250^{\circ} \mathrm{C}\right)$. Alternating supply epitaxy (ASE) was employed to grow the single crystal 3C-SiC(1 00 ) $/ \mathrm{Si}\left(\begin{array}{lll}1 & 0 & 0\end{array}\right)$ and the precursors $\mathrm{SiH}_{4}$ and $\mathrm{C}_{3} \mathrm{H}_{6}$ were employed as source of $\mathrm{Si}$ and $\mathrm{C}$ atoms. The grown films were unintentionally doped with n-type conductance. The full details of the growth process can be found in [23,24]. After the growth process, X-ray diffraction (XRD) analysis of the grown films was carried out to confirm the crystal structure followed by the rocking curve to analyze the crystalline quality. Fig. 1(a) shows the XRD pattern of n-type 3C-SiC( $\left.\begin{array}{lll}1 & 0 & 0\end{array}\right)$ thin film on $\operatorname{Si}\left(\begin{array}{lll}1 & 0 & 0\end{array}\right)$ in conventional $\theta-2 \theta$ scan mode. It can be confirmed from Fig. 1(a) that only the peaks corresponding to $\left(\begin{array}{lll}1 & 0 & 0\end{array}\right)$ plane are present which confirms that single crystal 3C-SiC( $\left.\begin{array}{lll}1 & 0 & 0\end{array}\right)$ was grown on $\mathrm{Si}\left(\begin{array}{lll}1 & 0 & 0\end{array}\right)$. Fig. 1(b) shows the rocking curve of $3 \mathrm{C}-\mathrm{SiC}\left(\begin{array}{lll}1 & 0 & 0\end{array}\right)$ peak and the observed full width at half maximum (FWHM) value is $0.70^{\circ}$ which shows the good crystalline quality of the grown film. Atomic force microscopy (AFM) was used to measure the roughness of the grown thin films. The AFM images of n-type 3C-SiC( $\left(\begin{array}{ll}1 & 0\end{array}\right)$ thin film for a scan area of $5 \mu \mathrm{m} \times 5 \mu \mathrm{m}$ is shown in Fig. $1(\mathrm{c})(\mathrm{c})$. The root mean square (RMS) roughness of n-type 3C-SiC( $\left.\begin{array}{lll}1 & 0 & 0\end{array}\right)$ was measured to be $2 \pm 0.5 \mathrm{~nm}$ which shows the excellent surface morphology of the grown films. The conductance type and carrier concentration of the grown thin film was carried out by hot probe measurements and then confirmed by Hall effect measurements. The carrier concentration of n-type single crystalline 3C-SiC(1 00 ) was found to be $5-8 \times 10^{16} \mathrm{~cm}^{-3}$. The carrier concentration of the Si substrates was $5 \times 10^{14} \mathrm{~cm}^{-3}$. The electrical resistivity was found to be 1.4 $\Omega \mathrm{cm}$ with corresponding electron mobility of $60 \mathrm{~cm}^{2} / \mathrm{vs}$.

Square shaped Hall devices were fabricated in different crystal orientations using conventional photolithography and dry etch processes (Fig. 2(a)) to investigate the stress-induced pseudo-Hall effect. The square shape is chosen for the study because it produces maximum pseudo-Hall effect [16]. After fabrication of the devices, the wafer was diced into strips with dimensions of $60 \mathrm{~mm} \times 9 \mathrm{~mm} \times 0.625 \mathrm{~mm}$ to apply stress by the bending beam method. Cantilever method was used to apply the stress to the devices in which one end of the beam with the devices was fixed, while a known force was applied to the other end. Consequently stresses from 0 to $343 \mathrm{MPa}$ were induced to n-type 3C-
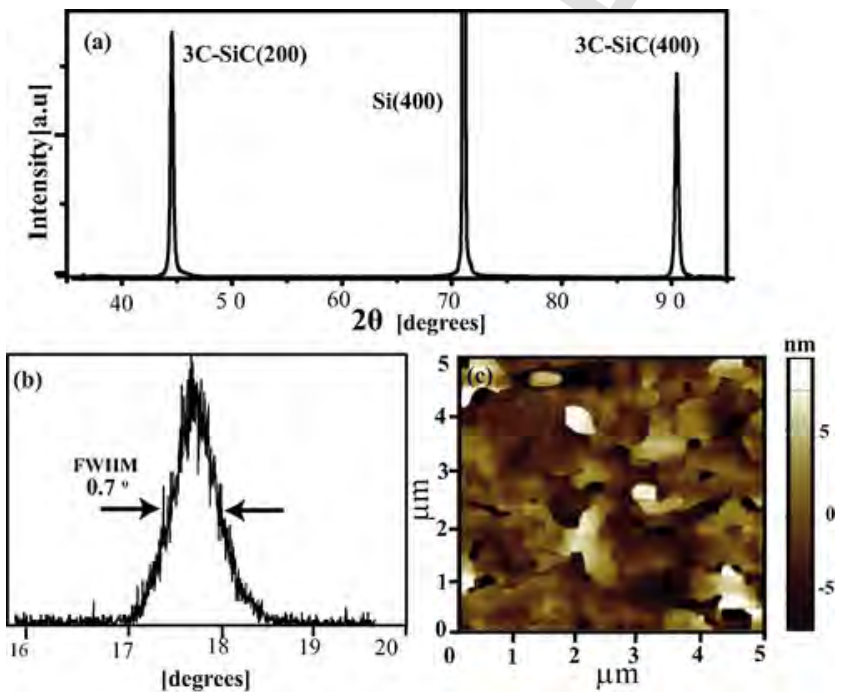

Fig. 1. (a) XRD pattern of single crystal n-type $3 C$-SiC( 100 ), (b) The rocking curve of 3C-SiC(1 00 ), (c) AFM image of n-type 3C-SiC( $\left.\begin{array}{lll}1 & 0 & 0\end{array}\right)$ showing a high quality thin film.
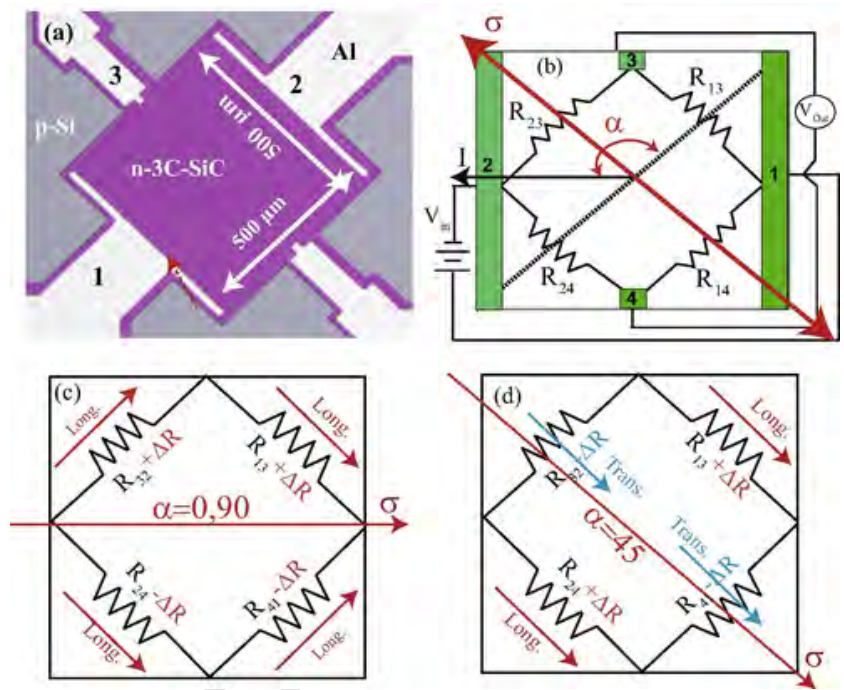

Fig. 2. (a) Microscope image of the fabricated Hall device, (b) equivalent circuit diagram of the device, (c) counter balance in Change of resistances under stress when $\alpha=0^{\circ}, 90^{\circ}$, (d) Observation of maximum pseudo-Hall effect when $\alpha=45^{\circ}$.

SiC layer to investigate the pseudo-Hall effect . The method to numerically calculate the stress-induced into the 3C-SiC layer on Si strip is reported elsewhere [22,?]. The Ohmic contacts were confirmed by $I-V$ measurement and horizontal and vertical current leakages from n-type 3C-SiC( $\left.\begin{array}{lll}1 & 0 & 0\end{array}\right)$ through to the $\mathrm{Si}$ substrate were measured to be less than $2 \%$ of the total device current. Therefore, the current leakage is negligible and it does not contribute in the measurements.

\section{Results and discussion}

For the measurement of pseudo-Hall effect the current was applied at terminal 1 and 2 (Fig. 2(a)) while the output voltage was recorded at terminals 3 and 4 . The equivalent circuit diagram of the device is shown in Fig. 2(b) while the Fig. 2(c, d) explain the different configurations of applied stress and current. When the stress is applied to the device at a constant input current the offset voltage at terminals 3 and 4 changes corresponding to the change in applied stress which is called the pseudo-Hall effect. Fig. 3(a) shows the pseudo-Hall effect in n-type 3C-SiC(1 00 ) thin film when the applied stress is in $\left[\begin{array}{lll}1 & 0 & 0\end{array}\right]$ direction. The device behavior was investigated at three different direction of applied current (i.e. $\left.\alpha=0^{\circ}, 90^{\circ}, 45^{\circ}\right)$ in $\left(\begin{array}{lll}1 & 0 & 0\end{array}\right)$ plane as shown in Fig. 3.

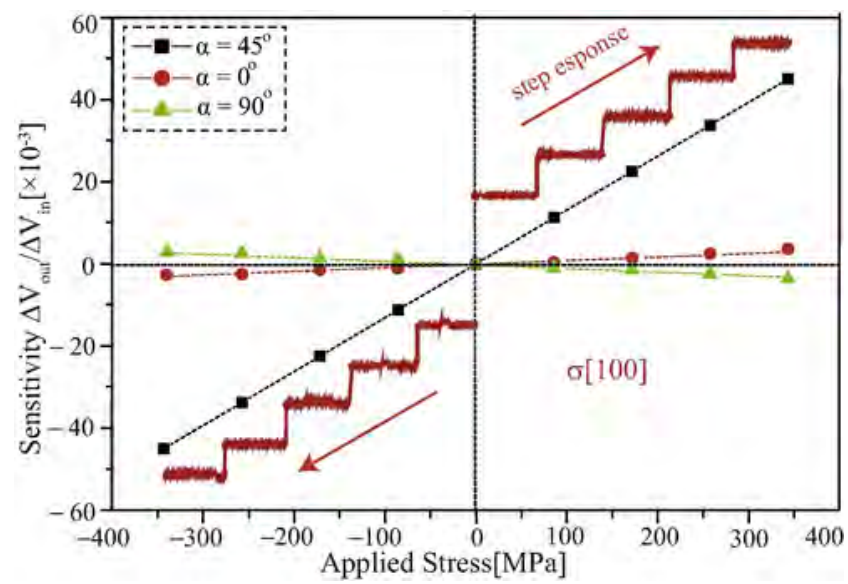

Fig. 3. The change in ratio of output voltage to the input voltage when stress is applied in $\left[\begin{array}{lll}1 & 0 & 0\end{array}\right]$ direction with different directions of input current. The stair like structure in the inset shows the output response of the device recorded on oscilloscope by applying the load in steps. 
For the sake of simplicity the pseudo-Hall effect is represented as the change in ratio of the output voltage to the input voltage of the pseudo-Hall device with change in applied stress. It can be observed from Fig. 3 that the ratio of the output voltage to the input voltage shows a smooth linear behavior to the applied compressive and tensile stresses and it varies significantly as the direction of device current is rotated in $\left(\begin{array}{lll}1 & 0 & 0\end{array}\right)$ crystal plane. The device has highest pseudo-Hall effect, when the current is in $\left[\begin{array}{lll}1 & 1 & 0\end{array}\right]$ direction and the applied stress is in $\left[\begin{array}{lll}1 & 0 & 0\end{array}\right]$ direction. The pseudo-Hall effect for other direction of input current is negligible. The stair-like behavior shown in Fig. 3 is the oscilloscope response of the device at different loads applied in steps.

Fig. 4 shows the pseudo-Hall effect in n-type 3C-SiC( $\left.\begin{array}{lll}1 & 0 & 0\end{array}\right)$ Hall device when the stress in applied in $\left[\begin{array}{lll}1 & 1 & 0\end{array}\right]$ direction. Similar to the previous case the direction of device current was varied and the pseudo-Hall effect was observed for three different directions of the applied current (i.e. $\alpha=0^{\circ}, 90^{\circ}, 45^{\circ}$ ). It is very clear form Fig. 1 that the pseudo-Hall effect also shows a smooth linear behavior with applied stress for this configuration but it is almost 10 times smaller than the previous configuration. Therefore, the pseudo-Hall effect in n-type 3C-SiC(1 00 0 $)$ strongly depends upon the crystal orientation. It is important to note that the pseudo-Hall effect in n-type 3C-SiC(llllll 00 shows completely different dependence on crystallographic orientation as compared to p-type 3C-SiC( $\left.\begin{array}{lll}1 & 0 & 0\end{array}\right)$ thin film. Moreover, the pseudo-Hall effect in n-type 3C-SiC(1 00 ) with low carrier concentration investigated in this study is $50 \%$ larger than the maximum pseudo-Hall effect observed in p-type 3C-SiC(1 00 ) reported in [19].

The origin of this effect can be explained by using a equivalent circuit model presented in Fig. 2(b). From the equivalent circuit shown in Fig. 2(b), the offset voltage $V_{o}$ can be presented using a square type bridge circuit consisting of $R_{13}, R_{14}, R_{23}$ and $R_{24}$; and without stress $R_{13}=R_{14}=R_{23}=R_{24}=R_{\text {in }}$, where $R_{\text {in }}$ is initial resistance without stress). The output voltage of the device is given by [1]:

$V_{o}=\frac{\left(R_{13} R_{24}-R_{14} R_{23}\right) V_{i n}}{\left(R_{14}+R_{24}\right)\left(R_{13}+R_{23}\right)}$

When stress is applied to the square device, the resistors of the bridge circuit model change their value due to piezoresistive effect. Depending upon the direction of applied stress these changes in resistances of the individual resistors may counter balance each other or they can disturb the symmetry of the device leading to change in offset voltage. For example when the direction of input current is along the direction of applied stress or perpendicular $\left(\alpha=0^{\circ}, 90^{\circ}\right)$ each resistor experience longitudinal stress and change in resistances is in same direction which counter balance each other and hence small or no

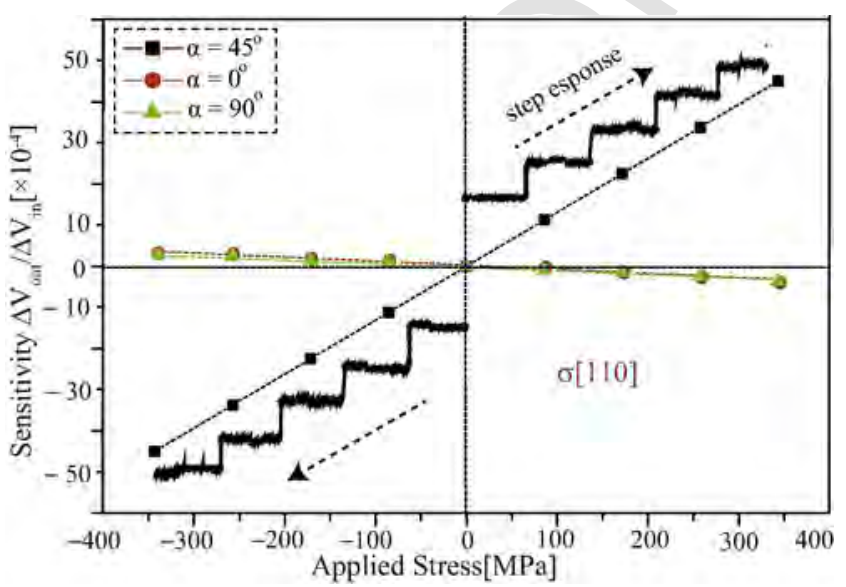

Fig. 4. The change in ratio of output voltage to the input voltage when stress is applied in $\left[\begin{array}{lll}1 & 1 & 0\end{array}\right]$ direction with different directions of input current. The stair like structure in the inset shows the output response of the device recorded on oscilloscope by applying the load in steps.
pseudo-Hall effect is observed (Fig. 2(c)). But when the direction of current is at $\left(\alpha=45^{\circ}\right.$ ) resistors $R_{14}$ and $R_{23}$ are stressed in longitudinal direction, whereas resistors $R_{13}$ and $R_{24}$ stressed in transverse direction, leading to a change in the symmetry of the device (Fig. 2(d). This results in a shift of offset voltage $V_{o}$. The change of these resistance under stress $\sigma$ are [1]:

$\left\{\begin{array}{l}R_{13}=R_{24}=R_{i n}\left[1+\left(\pi_{l} \cos ^{2} \alpha+\pi_{t} \sin ^{2} \alpha\right) \sigma\right] \\ R_{23}=R_{14}=R_{\text {in }}\left[1+\left(\pi_{t} \cos ^{2} \alpha+\pi_{l} \sin ^{2} \alpha\right) \sigma\right]\end{array}\right.$

where $\pi_{l}$ and $\pi_{t}$ are longitudinal and transverse piezoresistive coefficients of n-type $3 \mathrm{C}$-SiC with low carrier concentration in the direction of applied current which can be calculated by the method presented in appendix A. $\alpha$ is the angle between direction of stress and diagonal of the device indicated by dotted line in Fig. 2(b). From Eqs. (1) and (2), the generated output voltage $\left(V_{0}\right)$ at terminals 3 and 4 when applying input voltage $\left(V_{\text {in }}\right)$ at terminals 1 and 2 is calculated as:

$V_{0}=\frac{\left(\cos ^{2} \alpha-\sin ^{2} \alpha\right)\left(\pi_{l}-\pi_{t}\right) \sigma V_{i n}}{2}$

Table 1 shows the comparison of pseudo-Hall effect for different 3C-SiC thin films grown by LPCVD process for different directions of applied current and stress. It can be observed from Table 1 that the n-type 3C-SiC( $\left.\begin{array}{lll}1 & 0 & 0\end{array}\right)$ has the maximum pseudo-Hall effect when the direction of current is $\left[\begin{array}{lll}1 & 1 & 0\end{array}\right]$ and the direction of applied stress is in $\left[\begin{array}{lll}1 & 0 & 0\end{array}\right]$ direction. The pseudo-Hall effect is $50 \%$ larger than the maximum pseudo-Hall effect observed in p-type 3C-SiC( $\left.\begin{array}{lll}1 & 0 & 0\end{array}\right)$ with higher carrier concentration. Additionally, the n-type 3C-SiC( $\left.\begin{array}{lll}1 & 0 & 0\end{array}\right)$ shows strong dependence on crystallographic orientation which is completely different from the results obtained in p-type 3C-SiC(1 00 ) thin films prepared by the same process. This different behavior of the pseudo-Hall effect can be attributed to the different conduction mechanisms in p-type and n-type 3C-SiC( $\left.\begin{array}{lll}1 & 0 & 0\end{array}\right)$. In case of p-type 3C-SiC, the holes are distributed in light and heavy hole sub-bands in valence band. When stress is applied to p-type 3C-SiC, the light hole and heavy hole bands split and hole re-distribution occurs, leading to change in conductance mechanism and ultimately the pseudo-Hall effect. For n-type 3C-SiC, the electrons are distributed in 6 equivalent energy valleys. When stress is applied, the electrons repopulate due to energy level shifts, leading to change in electron effective mass and mobility which ultimately results in change of pseudo-Hall effect. As low doped n-type $3 \mathrm{C}-\mathrm{SiC}$ is also used for Hall sensors which require minimum pseudo-Hall effect. Therefore, the least affected crystal orientation by pseudo-Hall effect is also presented in this study which is very helpful in the designing of Hall sensors for magnetic field sensing.

\section{Conclusion}

In conclusion the pseudo-Hall effect for single crystal n-type 3C-SiC(1 00 ) with low carrier concentration has been investigated for different crystallographic orientations. It has been found that unlike p-type 3C-SiC( $\left.\begin{array}{lll}1 & 0 & 0\end{array}\right)$ pseudo-Hall effect shows completely different orien-

Table 1

Comparison of the pseudo-Hall effect in different types of 3C-SiC thin films prepared by LPCVD process reported to date:the applied stress was fixed at $264 \mathrm{MPa}$. [16,17]

\begin{tabular}{|c|c|c|c|c|}
\hline $\begin{array}{l}\text { Type of } \\
\text { SiC }\end{array}$ & $\begin{array}{l}\text { Crystal } \\
\text { plane }\end{array}$ & $\begin{array}{l}\text { Stress } \\
\text { direction }\end{array}$ & $\begin{array}{l}\text { Current } \\
\text { direction }\end{array}$ & $\begin{array}{l}\Delta V_{\text {out }} / \Delta V_{\text {in }} \\
\left.10^{-3}\right]\end{array}$ \\
\hline *n-type & $\left(\begin{array}{lll}1 & 0 & 0\end{array}\right)$ & {$\left[\begin{array}{lll}1 & 0 & 0\end{array}\right]$} & {$\left[\begin{array}{lll}1 & 1 & 0\end{array}\right]$} & 47.5 \\
\hline$*$ n-type & $\left(\begin{array}{lll}1 & 0 & 0\end{array}\right)$ & {$\left[\begin{array}{lll}1 & 1 & 0\end{array}\right]$} & {$\left[\begin{array}{lll}1 & 0 & 0\end{array}\right]$} & 4.8 \\
\hline p-type & $\left(\begin{array}{lll}1 & 0 & 0\end{array}\right)$ & {$\left[\begin{array}{lll}1 & 0 & 0\end{array}\right]$} & {$\left[\begin{array}{lll}1 & 1 & 0\end{array}\right]$} & 3.5 \\
\hline p-type & $\left(\begin{array}{lll}1 & 0 & 0\end{array}\right)$ & {$\left[\begin{array}{lll}1 & 1 & 0\end{array}\right]$} & {$\left[\begin{array}{lll}1 & 0 & 0\end{array}\right]$} & 24 \\
\hline p-type & $\left(\begin{array}{lll}1 & 1 & 1\end{array}\right)$ & {$\left[\begin{array}{lll}1 & 1 & 0\end{array}\right]$} & {$[-]$} & 4.81 \\
\hline p-type & $\left(\begin{array}{lll}1 & 1 & 1\end{array}\right)$ & {$\left[\begin{array}{lll}1 & 1 & \overline{2}\end{array}\right]$} & {$[-]$} & 4.7 \\
\hline
\end{tabular}

* Present study. 
tation dependence in n-type 3C-SiC( $\left.\begin{array}{lll}1 & 0 & 0\end{array}\right)$ with low carrier concentration. The pseudo-Hall effect is maximum for applied stress in $\left[\begin{array}{lll}1 & 0 & 0\end{array}\right]$ and applied current in $\left[\begin{array}{lll}1 & 1 & 0\end{array}\right]$ direction. The values of psudo-Hall effect in n-type 3C-SiC(1 00 ) is 50\% larger than the pseudo-Hall effect in p-type 3C-SiC( $\left.\begin{array}{lll}1 & 0 & 0\end{array}\right)$ which makes n-type 3C-SiC( $\left.\begin{array}{lll}1 & 0 & 0\end{array}\right)$ with low carrier concentration more suitable material for stress sensing applications.

\section{Acknowledgements}

This work was performed in part at the Queensland node of the Australian National Fabrication Facility, a company established under the National Collaborative Research Infrastructure Strategy to provide nano and micro-fabrication facilities for Australia's researchers. This work has been partially supported by the Griffith University's New Researcher Grants.

\section{References}

[1] Y. Kanda, A. Yasukawa, Sens. Actuators 2 (1982) 283

[2] Y. Kanda, K. Yamamura, Sens. Actuators 18 (1989) 247.

[3] J.C. Doll, B.L. Pruitt, Piezoresistor Design and Applications, Springer, New York, 2013.

[4] J. Gragg, Silicon pressure sensor, Patent US 4317126, 1982.

[5] A. Mian, J.C. Suhling, R. Jaeger, IEEE Sensors J. 6 (2006) 340-356.

[6] Y. Kanda, Jpn. J. Appl. Phys. 26 (1987) 1031.
[7] A.V. Gridchin, V.A. Gridchin, Sens. Actuators A 58 (1997) 219.

[8] M. Doelle, D. Mager, P. Ruther, O. Paul, Sens. Actuators A 127 (2006) 261

[9] Y. Kanda, M. Migitaka, Phys. Status Solidi A 35 (1976) K115.

[10] Y. Kanda, M. Migitaka, Phys. Status Solidi A 38 (1976) K41.

[11] A. Qamar, P. Tanner, D.V. Dao, H.-P. Phan, T. Dinh, IEEE Electron Device Lett. 35 (2014) 1293-1295.

[12] A. Qamar, D.V. Dao, P. Tanner, H.-P. Phan, T. Dinh, S. Dimitrijev, IOP Appl. Phys. Express 8 (2015) 061302.

[13] A. Qamar, D.V. Dao, H.-P. Phan, T. Dinh, S. Dimitrijev, Appl. Phys. Lett. 109 (2016) 092903.

[14] A. Qamar, H.-P. Phan, T. Dinh, L. Wang, S. Dimitrijev, D.V. Dao, RSC Adv. 6 (2016) 31191-31195.

[15] H.P. Phan, D.V. Dao, P. Tanner, N.T. Nguyen, J.S. Han, S. Dimitrijev, G. Walker, L. Wang, Y.J. Zhu, Matter. Chem. C 2 (2014) 7176-7179.

[16] H.P. Phan, D.V. Dao, L. Wang, T. Dinh, N.-T. Nguyen, A. Qamar, P. Tanner, S. Dimitrijev, Y.J. Zhu, Matter. Chem. C 3 (2015) 1172-1176.

[17] S. Roy, C. Jacob, S. Basu, IEEE Trans. Electron Devices 94 (2003) 298.

[18] A. Qamar, H.-P. Phan, D. Dao, P. Tanner, T. Dinh, L. Wang, S. Dimitrijev, IEEE Electron Device Lett. (2015) 1.

[19] A. Qamar, H.-P. Phan, J. Han, P. Tanner, T. Dinh, L. Wang, S. Dimitrijev, D.V.J. Dao, Mater. Chem. C 3 (2015) 8804-8809.

[20] A. Qamar, D.V. Dao, J. Han, H.-P. Phan, A. Younis, P. Tanner, T. Dinh, L. Wang, S.J. Dimitrijev, Mater. Chem. C 3 (2015) 12394-12398.

[21] A. Iacopi, D.V. Dao, S. Dimitrijev, J.S. Han, A. Qamar, T. Dinh, H.P. Phan, Pseudo-Hall effect in single crystal n-type 3C-SiC(100) thin film, Mater. Technol. Modern Indus. Prod. (2017) 3-7.

[22] H.-P. Phan, A. Qamar, D.V. Dao, T. Dinh, L. Wang, J. Han, P. Tanner, S. Dimitrijev, N.-T. Nguyen, RSC Adv. 5 (2015) 56377-56381.

[23] L. Wang, A. Iacopi, S. Dimitrijev, G. Walker, A. Fernandes, L. Hold, J. Chai, Thin Solid Films 564 (2014) 39-44.

[24] L. Wang, S. Dimitrijev, J. Han, P. Tanner, A. Lacopi, L. Hold, J. Cryst. Growth 329 (2011) 67-70. 